\title{
Hydroelastic solitary waves with constant vorticity
}

\author{
Tao Gao ${ }^{\mathrm{a}, *}$, Paul Milewski ${ }^{\mathrm{a}}$, Jean-Marc Vanden-Broeck ${ }^{\mathrm{b}}$ \\ ${ }^{a}$ Department of Mathematical Sciences, University of Bath, Bath BA2 7AY, UK \\ ${ }^{b}$ Department of Mathematics, University College London, London WC1E 6BT, UK
}

\begin{abstract}
In this work, two-dimensional hydroelastic solitary waves in the presence of constant vorticity are studied. Time-dependent conformal mapping techniques first developed for irrotational waves are applied subject to appropriate modification. An illustrative high-order Nonlinear Schrödinger Equation is presented to investigate whether a given envelope collapses into a singular point in finite time by using virial theory. Travelling solitary waves on water of infinite depth are computed for different values of vorticity and new generalised solitary waves are discovered. The stabilities of these waves are examined numerically by using fully nonlinear time-dependent computations which confirm the virial theory analysis.
\end{abstract}

Keywords: surface gravity waves, solitary waves, elastic waves, asymmetric waves

\section{Introduction}

The study of the deformation of floating ice sheets plays a major role in understanding the characteristics of waves propagating under ice cover in polar regions [1], in assuring the safety of human activities, such as transportation, over ice sheets [2], and in understanding the breakup of continuous sheets. Waves in such system, called hydroelastic or flexural-gravity waves, have therefore received extensive research interest (see $[2,3,4,5])$. In the simplest situation the ice sheet is modelled as a thin flexible but inextensible layer at the surface of the fluid. There are several models for this thin flexible layer. Euler-Bernoulli beam theory, a linear model of elasticity, was first

\footnotetext{
${ }^{*}$ Corresponding author

Email address: t.gao@bath.ac.uk (Tao Gao)
} 
used to compute the bending of the elastic sheet. However this theory is not applicable to the case of large deformation where a nonlinear model should be employed. Two nonlinear models have also been used. In the KirchhoffLove model, where the pressure exerted by the elastic sheet is expressed by the second derivative of the curvature of the surface with respect to the horizontal, was used to study periodic waves $([6,7])$, generalised solitary waves ([8]), dark solitary waves $([9])$ and solitary waves ([10]) in the absence of external forcing. On the other hand, the moving load problem was considered in $[10,11,12]$. More recently, Toland $[13,14]$ proposed a geometric nonlinear model using the Cosserat theory of hyperelastic shells, which takes the form

$$
\mathcal{P}=D\left(\kappa_{s s}+\frac{1}{2} \kappa^{3}\right)
$$

where $\mathcal{P}$ is the pressure jump across the sheet, $D$ is its flexural rigidity, $\kappa$ is the curvature of the sheet and $s$ is arc length. Some of the problems mentioned above were revisited in $[15,16,17,18]$ under this flexural model.

All the works mentioned in the last paragraph assumed that the fluid considered is inviscid, incompressible and irrotational. However, a theory with a nonzero vorticity is both of theoretical interest and may also be more physically realistic in certain cases. For example, in ice sheets over flowing water or over non-uniform currents, the irrotational assumption is invalid. Here, we consider the situation when the flow has constant vorticity, which is also viewed as the wave problem when uniform shear currents are taken into account.

Previously, classical water waves (i.e. gravity waves) with constant vorticity were investigated intensively. In particular, a rigorous analytical study was achieved by [19] (see this work and references therein for a review). On the numerical side, fully nonlinear steady state solutions with constant vorticity in these classical problems were computed by a boundary integral equation method in $[20,21,22,23,24,25]$. The dynamics of unsteady solutions, were studied using a time-dependent conformal mapping technique in [26]. Wave interactions in the same context were considered in [27]. The instability problem was studied recently in [28]. With the inclusion of capillary effects, steady and unsteady solutions were computed in [29] by using the same numerical techniques. In this work, we aim to investigate steady and unsteady hydroelastic waves in the presence of constant vorticity numerically by employing conformal mapping transformation.

While symmetric waves (i.e. waves for which there is a reflection symme- 
try about some point) have been extensively studied in fluid problems (see all references except $[16,34,35,36,38]$ and rigorous works $[30,31]$ for the irrotational case and $[32,33]$ for the rotational situation), there has been less work on asymmetric ones. They were first studied by [34] in the fifth order Korteweg de-Vries equation as a model for capillary-gravity. They show that multi-wavepacket asymmetric solitary waves can exist. Later fully nonlinear asymmetric solitary waves were numerically computed by [35] and [16] for the capillary-gravity and flexural-gravity problem respectively. Asymmetric generalised solitary waves, i.e. asymmetric solitary waves with ripples of constant amplitude in the far field, were discovered in [36] for the problem of capillary-gravity waves under the effect of normal electric fields by using a model equation resulting from a quintic truncation of the Dirichletto-Neumann operator [37]. In [38] fully nonlinear asymmetric generalised capillary-gravity solitary waves were found.

In this work, the Cosserat model (1) is used in modelling the bending of the elastic sheet at the free surface of a fluid of infinite depth. We find symmetric and asymmetric solitary waves for the fully nonlinear problem when the effect of constant vorticity is present. Time-dependent simulations are then performed in order to test the stability of solitary waves, and explore the wave interaction problem. The paper is structured as follows. In section 2 , the detailed mathematical formulation is stated. It is followed by a description of the numerical scheme in section 3. A reduced model is used to interpret the numerical results of the full Euler equations that are presented in section 4 and discussed in section 5 .

\section{Formulation}

We consider an inviscid and incompressible flow on a fluid with constant vorticity $\Omega$ of infinite depth whose upper surface is covered by an elastic sheet with flexural rigidity $D$. The deformation of the upper surface is described by a train of waves with wavelength $\lambda$ travelling at speed $c$. Two-dimensional Cartesian coordinates are used with positive $x$-direction pointing the direction in which the waves travel and negative $y$-direction following that of the gravity $g$. We denote the velocity by $(u, v)$ and unknown free surface by $y=\zeta(x, t)$. The mean flow level is set at $y=0$ and the pressure above the elastic sheet is zero. The jump in pressure across the sheet is given by (1). The vorticity can be expressed by

$$
\Omega_{0}=v_{x}-u_{y},
$$




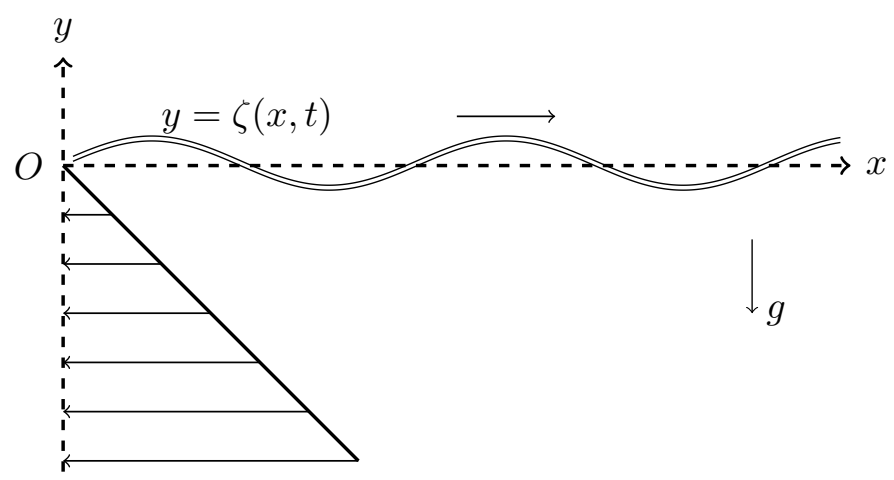

Figure 1: Configuration of the problem. $\Omega_{0}$ is negative.

where the subscripts denote the partial derivatives.

Now we introduce the streamfunction $\varphi$ and then we have

$$
\begin{aligned}
& u=\varphi_{y}, v=-\varphi_{x} \\
& \nabla^{2} \varphi=-\Omega_{0} .
\end{aligned}
$$

A modified streamfunction $\psi$ can be written by

$$
\psi=\varphi+\frac{\Omega_{0} y^{2}}{2}
$$

Then $\psi$ also satisfies the Laplace equation

$$
\nabla^{2} \psi=0
$$

Its harmonic conjugate $\phi$ can be defined in the following way

$$
\begin{aligned}
& \phi_{x}=\psi_{y}=u+\Omega_{0} y, \\
& \phi_{y}=-\psi_{x}=v .
\end{aligned}
$$

By definition, $\phi$ satisfies the Laplace equation in the flow domain. The governing equations are as follows

$$
\begin{array}{ll}
\nabla^{2} \phi=0, & y<\zeta(x, t), \\
\phi_{t}+\frac{1}{2}|\nabla \phi|^{2}+\Omega\left(\psi-\zeta \phi_{x}\right)+\zeta+\left(\kappa_{s s}+\frac{1}{2} \kappa^{3}\right)=0, & \text { on } y=\zeta(x, t), \\
\zeta_{t}=\phi_{y}-\left(\phi_{x}-\Omega \zeta\right) \zeta_{x}, & \text { on } y=\zeta(x, t), \\
\phi_{y}=0, & \text { as } y \rightarrow-\infty,
\end{array}
$$


where the system is non-dimensionalised by choosing

$$
\left(\frac{D}{\rho g}\right)^{\frac{1}{4}}, \quad\left(\frac{D}{\rho g^{5}}\right)^{\frac{1}{8}}, \quad\left(\frac{D^{3} g}{\rho^{3}}\right)^{\frac{1}{8}}
$$

as the reference length, time and stream scale with $\rho$ being the density of the fluid, $s$ being the arclength and $\kappa$ being the curvature. The rescaled vorticity $\Omega$ is defined by

$$
\Omega=\left(\frac{D}{\rho g^{5}}\right)^{\frac{1}{8}} \Omega_{0}
$$

Equations (10) and (11) are the dynamic and kinematic boundary conditions respectively on the free surface and (12) is the impermeability condition on the bottom. We note that, in the particular case where $\Omega=0, \phi$ is the standard velocity potential and (10) is the standard Bernoulli equation for flexural-gravity waves. The system is linearised by writing $\zeta=C_{1} e^{i k\left(x-c_{p} t\right)}$, $\phi=C_{2} e^{i k\left(x-c_{p} t\right)+|k| y}$ where $C_{1}$ and $C_{2}$ are small constants. Substituting into (12) and making use of (11) yields the linear dispersion relation

$$
c_{p}^{2}-\frac{\Omega}{|k|} c_{p}-\frac{1}{|k|}\left(1+k^{4}\right)=0
$$

where $c_{p}$ is the phase speed. This relation is invariant under the reflection $\left(c_{p}, k, \Omega\right) \rightarrow\left(-c_{p},-k,-\Omega\right)$. This means that if we are interested in studying unidirectional propagating waves we can choose $k>0$ and $c_{p}>0$, varying $\Omega$ to be positive or negative. The two branches of (15) for $k>0$ and varying $\Omega$ are shown in figure 2. As can be seen from the graph, the upper curve and the lower curve have different behaviour in the presence of a non-zero $\Omega$, and their role is interchanged when the sign of $\Omega$ is changed. Note that for bi-directional waves, the extrema of $c_{p}$ are attained at distinct values of $k$ so that solitary waves in opposite directions have different carrier wavenumbers.

\section{Numerical scheme}

\subsection{Time-evolutionary system}

To solve the fully nonlinear problem, a time-dependent conformal mapping, first pioneered by [39], is used to transform the flow domain in the

$x-y$ plane into a simple fixed region which is chosen to be the lower half 

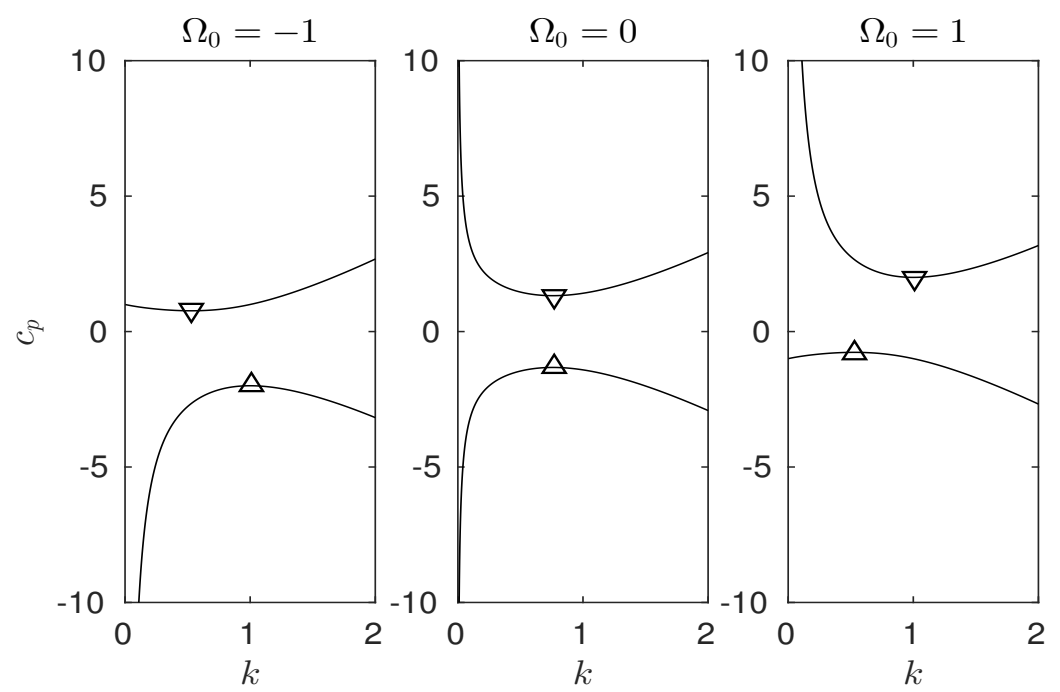

Figure 2: Linear dispersion of flexural-gravity waves with different values of vorticity $\Omega_{0}=-1, \Omega_{0}=0$ and $\Omega_{0}=1$ (from left to right). The extrema are marked as the triangles.

of the $\xi-\eta$ plane for the deep water case. The surface variables are defined by $Y(\xi, t)=y(\xi, 0, t), X(\xi, t)=x(\xi, 0, t), \Phi(\xi, t)=\phi(\xi, 0, t)$ and $\Psi(\xi, t)=\psi(\xi, 0, t)$. The explicit form of the conformal map from $(x, y)$ to $(\xi, \eta)$ can be found by solving the boundary value problem as follows

$$
\begin{array}{ll}
y_{\xi \xi}+y_{\eta \eta}=0, & \text { for } \eta<0, \\
y(\xi, 0, t)=Y(\xi, t), & \text { on } \eta=0, \\
y_{\eta}=1, & \text { on } \eta \rightarrow-\infty .
\end{array}
$$

The associate map for $x(\xi, \eta)$ is the harmonic conjugate of $y(\xi, \eta)$. This is useful in investigating the flow structure beneath the wave (see e.g. [42]) but is not required in studying the dynamics of the free surface. After performing similar calculations as those presented in [29, 40, 41], we have

$$
\begin{aligned}
& X_{\xi}=1-\mathcal{H}\left[Y_{\xi}\right], \\
& \Phi_{\xi}=-\mathcal{H}\left[\Psi_{\xi}\right],
\end{aligned}
$$

where the $\mathcal{H}$ is the Hilbert transform defined by

$$
\mathcal{H}[f](\xi)=\int \frac{f\left(\xi^{\prime}, 0, t\right)}{\xi^{\prime}-\xi} d \xi^{\prime} .
$$


In computations we shall use the fact that the Fourier symbol of $\mathcal{H}$ is $i \operatorname{sgn}(k)$.

Rewriting the governing equations in the mapped plane we obtain

$$
\begin{aligned}
Y_{t} & =Y_{\xi} \mathcal{H}\left[\frac{\Psi_{\xi}-Y Y_{\xi}}{J}\right]-X_{\xi}\left(\frac{\Psi_{\xi}-\Omega Y Y_{\xi}}{J}\right), \\
\Phi_{t} & =\frac{\Psi_{\xi}^{2}-\Phi_{\xi}^{2}}{2 J}-Y-\Omega\left(\Psi-\frac{Y X_{\xi} \Phi_{\xi}}{J}\right)+\Phi_{\xi} \mathcal{H}\left[\frac{\Psi_{\xi}-\Omega Y Y_{\xi}}{J}\right]-\mathcal{P},
\end{aligned}
$$

where $J=X_{\xi}^{2}+Y_{\xi}^{2}$ is the Jacobian of the mapping and the rescaled pressure $\mathcal{P}$ exerted by the elastic sheet is

$$
\mathcal{P}=\frac{1}{2}\left[\frac{\kappa_{\xi \xi}}{J}+\left(\frac{\kappa_{\xi}}{J}\right)_{\xi}+\kappa^{3}\right] .
$$

Equations (19), (20), (22) and (23) complete the time-evolutionary system. We now choose a Fourier spectral discretisation on the $\xi$ dependence. All derivatives and Hilbert transforms are evaluated spectrally in the Fourier space while the nonlinear terms are computed pseudo-spectrally in real space with dealiasing. A fourth-order Runge-Kutta method is used to integrate in time. Typical choices are $d \xi=0.05 \& d t=0.0005$ as the spatial and time step size for time-dependent computations.

\subsection{Travelling waves}

In order to compute travelling waves with wave speed $c$, we repeat the calculations above seeking solutions that depend on $x-c t$. The kinematic boundary condition (22) and the dynamic boundary condition (23) then reduce to

$$
\begin{aligned}
& \Psi_{\xi}=c Y_{\xi}+\Omega Y Y_{\xi}, \\
& Y+\frac{\left(c+\Omega Y X_{\xi}+\Omega \mathcal{H}\left[Y Y_{\xi}\right]\right)^{2}}{2 J}-\frac{c^{2}}{2}+\mathcal{P}=0 .
\end{aligned}
$$

We note that these formulations allows us to compute multi-valued profiles, i.e. overhanging waves.

The Hamiltonian of the system can be written by

$$
\mathcal{E}=\frac{1}{2} \int\left(Y^{2} X_{\xi}+\Psi \Phi_{\xi}+\frac{\Omega^{2}}{3} Y^{3} X_{\xi}-\frac{\Omega}{2} Y^{2} \Phi_{\xi}+\frac{\left(Y_{\xi \xi} X_{\xi}-X_{\xi \xi} Y_{\xi}\right)^{2}}{J^{5 / 2}}\right) d \xi,
$$

which will be used to numerically compute the energy of the solutions. The Hamiltonian formulations in the context of irrotational hydroelastic 
waves can also be used as an alternative approachto derive the Nonlinear Schrödinger Equation (see e.g. [18]) which will be discussed in section 4.1. We assume that waves are periodic with wavelength $L$ so we may represent $Y$ as a Fourier Series

$$
Y=\sum_{n=0}^{N} a_{n} \cos \left(\frac{2 n \pi \xi}{L}\right)+\sum_{n=0}^{N} b_{n} \sin \left(\frac{2 n \pi \xi}{L}\right),
$$

where the series is truncated after $N$ terms and the Fourier coefficients are the unknowns to be solved. We note that $b_{n}$ are all zeros in the case where the solution is symmetric by $x=0$. $L$ is taken to be sufficiently large for computing solitary waves such that a further increase in the wavelength does not change the solution. $L=79 \pi$ is intensively used in the computations. The amplitude $a$ is defined by

$$
a=\frac{1}{2}\left(\max _{\xi \in \mathbb{R}} Y-\min _{\xi \in \mathbb{R}} Y\right) .
$$

By introducing $N$ collocation points evenly distributed on the $\xi$-axis where the dynamic boundary conditions are satisfied, this nonlinear system with $N$ unknowns and $N$ equations can be solved by Newton's method. The required stopping criterion is when the $l^{\infty}$-norm of the residual error is less than $10^{-10}$.

\section{Results}

We present in section 4.1 the analyses based on the reduced models, namely a cubic and a quintic NLS, and follow this in section 4.2-4.4 with numerical results of the full Euler equations.

\subsection{Nonlinear Schrödinger Equation and virial Theory}

The bifurcation of small amplitude wave-packet solitary waves with a carrier wavenumber $k$ requires (i) an extrema in phase speed at $k=k^{*}$ and (ii) that the associated Nonlinear Schrödinger Equation (NLS) be of the focussing type at $k^{*}$ [43]. On the other hand, if the NLS is of defocussing type, wave-packet type solitary waves may bifurcate from finite amplitude as is the case for flexural-gravity waves in the absence of vorticity [16, 18, 47]. In general, the NLS equation for the evolution of a wavepacket $\eta=$ $\epsilon\left[A(\tau, X) e^{i k^{*}\left(x-c_{p} t\right)}+A^{*} e^{-i k^{*}\left(x-c_{p} t\right)}\right]$ has the form

$$
i A_{\tau}+\lambda A_{X X}+\gamma|A|^{2} A=0,
$$


where, $\tau=\epsilon^{2} t, X=\epsilon\left(x-c_{g} t\right), c_{g}$ is the group velocity and where the dispersion parameter $\lambda(k, \Omega)$ and the nonlinear parameter $\gamma(k, \Omega)$ determines the type of equation with $\lambda \gamma>0$ corresponding to the focussing case. For solitary wave bifurcations we need only consider wavenumbers for which $c_{p}$ is an extremum (i.e. $c_{p}^{\prime}\left(k^{*}\right)=0$ implying $c_{p}=c_{g}$ ), and it is easy to show that $\lambda\left(k^{*}, \Omega\right)>0$. Thus the one parameter function $\gamma\left(k^{*}, \Omega\right) \equiv \gamma^{*}(\Omega)$ defines whether the NLS is focussing type. From [48], it is found that $\gamma^{*}(0) \approx$ -0.0067 and that $\gamma^{*}<0$ for $\Omega>\Omega^{*}$ and $\gamma^{*}>0$ for $\Omega<\Omega^{*}$ where $\Omega^{*} \approx$ -0.0076 . From this we can conclude that for $\Omega<\Omega^{*}$ we expect solitary waves to bifurcate from the trivial state, whereas for $\Omega>\Omega^{*}$ they may bifurcate at finite amplitude (see the $\Omega=0$ case [47]). Furthermore, for values of vorticity near $\Omega^{*}, \gamma^{*}$ is very close to zero and higher order nonlinear effects become important. In this case a (simplified) higher order toy model could be proposed

$$
i A_{\tau}+\lambda A_{X X}+\gamma|A|^{2} A+\delta|A|^{4} A=0,
$$

a cubic-quintic NLS obtainable under appropriate redefinitions of the scalings for $\tau, X, A$. $\delta$ is the coefficient of the quintic nonlinear term which can be neglected when $\gamma$ is not close to zero. It corresponds to a higher order Stokes-wave frequency correction. This is a toy model because other terms are usually present at this order, but they don't change the scaling arguments below. Solitary waves for the equation above have the envelope satisfying

$$
\frac{1}{2} \lambda \rho_{X}^{2}+\frac{1}{2} \Delta C \rho^{2}+\frac{1}{4} \gamma \rho^{4}+\frac{1}{6} \delta \rho^{6}=0,
$$

where $\Delta C=c-c_{\text {min }}$ is the difference in wave speed from linear waves of minimum speed, and $\rho=|A|$. Periodic or solitary waves exist when the equation found by setting $\rho_{X}=0$ has a solution

$$
\Delta C+\frac{1}{2} \gamma \rho_{\max }^{2}+\frac{1}{3} \delta \rho_{\max }^{4}=0
$$

where we have used $\rho_{\max }$ to denote the amplitude of the periodic wave or wavepacket soliton. Fixing $\delta>0$, and assuming $\gamma$ is negative and small (i.e. near $\Omega=\Omega^{*}$ ), the behaviour of $\rho_{\max }$ is presented in figure 3 . When $\Delta C>0$ one expects to see periodic waves (or generalised solitary waves) whereas when $\Delta C<0$, one expects solitary waves, as the nonlinear solution has speed outside the linear spectrum. Finding $\rho_{\max }$ when $\Delta C=0$ and $\Delta C$ 


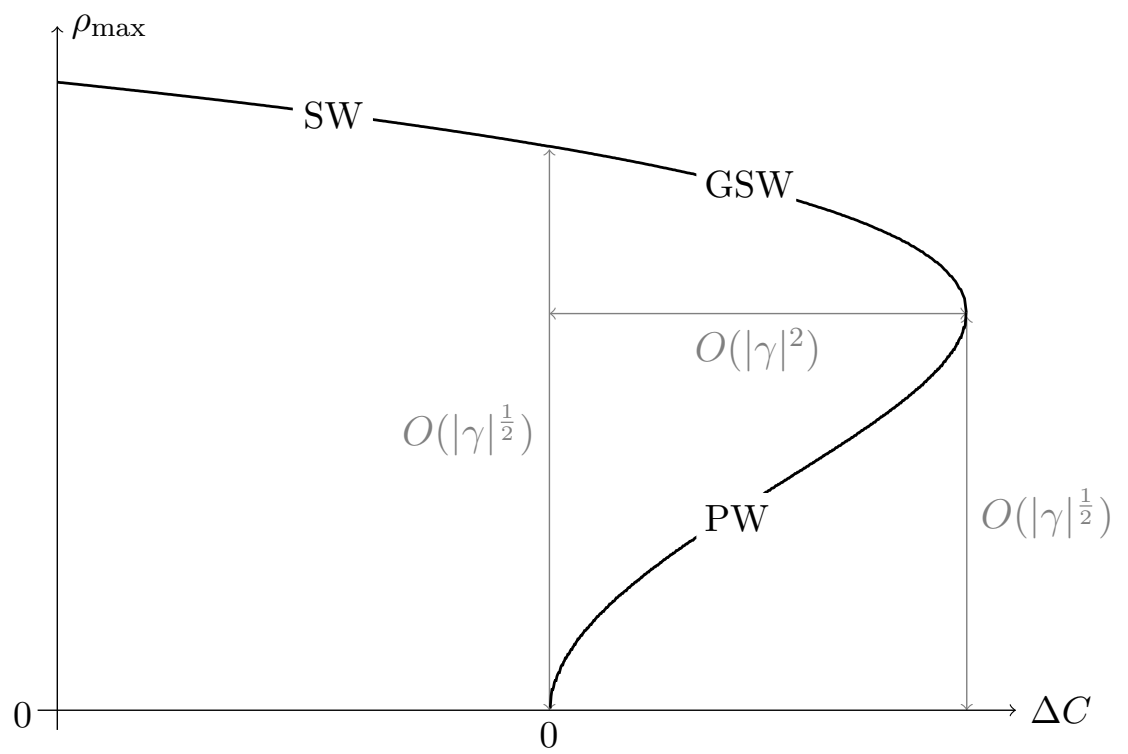

Figure 3: Graph of $\rho_{\max }$ against $\Delta C$ when $|\gamma|$ is small. The horizontal scale is exaggerated for a better display. SW, GSW, PW stand for solitary waves, generalised solitary waves and periodic waves respectively.

at the turning point of the amplitude-speed curve in (33) we find that

$$
\begin{array}{ll}
\rho_{\max }=O\left(|\gamma|^{\frac{1}{2}}\right), & \text { at the point where solitary waves emerge, } \\
\Delta C=O\left(|\gamma|^{2}\right), & \text { at the turning point of } \Delta C \text { - } \rho_{\max } \text { curve. }
\end{array}
$$

These are shown in figure 3. Therefore, for flexural-gravity waves in the absence of vorticity where $\gamma$ is close to zero, the width of region for generalised solitary waves is $O\left(|\gamma|^{2}\right)$, which is very narrow. It is numerically difficult to find these solutions with oscillatory tails in the far field by continuing the solution branch beyond $c_{\text {min }}$, as they quickly become Stokes Waves. It is much easier to find generalised solitary waves in the presence of a sufficiently strong positive vorticity which ensures $\gamma<0$ is not close to zero. For $\Omega<\Omega^{*}, \gamma>0$ and we do not expect to find generalised solitary waves which is confirmed by fully nonlinear numerical computations.

virial theory may be used to investigate whether a given initial envelope collapses into a singular point in finite time [45, 46]. In the context of the Euler equations this collapse was discussed in [44] for capillary-gravity waves. This theory can be applied to the current problem by using the cubic-quintic 
model (31) with the nonlinear term written as $f\left(|A|^{2}\right) A=\gamma|A|^{2} A+\delta|A|^{4} A$. We denote by $F$ the primitive function of $f$ and fix $\delta>0$. The solution of equation (31) conserves the "mass"

$$
\mathcal{M}=\int|A|^{2} d X
$$

and the associated Hamiltonian of the model (31)

$$
\mathcal{H}=\int\left|A_{X}\right|^{2}-\frac{\gamma}{2}|A|^{4}-\frac{\delta}{3}|A|^{6} d X
$$

We define the moment of inertia in the frame moving with the centre of the wavepacket by

$$
\mathcal{V}=\int X^{2}|A|^{2} d X
$$

Its second derivative with respect to time (see e.g. [45]), is given by

$$
\mathcal{V}_{\tau \tau}=4(2 \mathcal{H}+\Lambda)
$$

where $\mathcal{H}$ is conserved and the time-dependent term $\Lambda$ is expressed by

$$
\Lambda=3 \int F\left(|A|^{2}\right) d x-\int f\left(|A|^{2}\right)|A|^{2} d x=\int \frac{\gamma}{2}|A|^{4} d x .
$$

In the critical case where $\gamma=0$, it can be seen from (39) that $\mathcal{V}_{\tau \tau}$ is completely time-independent and $\mathcal{V}$ takes the form of

$$
\mathcal{V}=4 \mathcal{H} \tau^{2}+B \tau+C
$$

where $B=\mathcal{V}_{\tau}(\tau=0)$ and $C=\mathcal{V}(\tau=0)$ are the initial conditions. This means that for $\mathcal{H}<0$, then $\mathcal{V} \rightarrow 0$ in finite time. Due to the conservation of $\mathcal{M}$, we can deduce that the amplitude of the envelope $|A| \rightarrow 0$ almost everywhere except at the centre of mass where $|A| \rightarrow \infty$, i.e. the wave packet collapses into a single point in finite time. For $\mathcal{H}>0$, however, $\mathcal{V}$ increases without bound corresponding to the dispersive spreading of the wavepacket. A solitary wave solution $A=\mathcal{S}(X)$ satisfies $\mathcal{H}=0$. Applying a small perturbation of size $\epsilon$ to the ground state as $\tilde{\mathcal{S}}=(1+\epsilon) \mathcal{S}$, we substitute this form back into the Hamiltonian and obtain the following equation after elementary calculations

$$
\mathcal{H}(\tilde{\mathcal{S}})=-4 \epsilon\left|\mathcal{S}_{X}\right|^{2}+O\left(\epsilon^{2}\right) .
$$

Hence the virial theory predicts that a negative $\epsilon$ perturbation leads to an instability of wave spreading whereas a positive $\epsilon$ perturbation leads to wave collapse. Similar arguments can be made for $\gamma \neq 0$. 


\subsection{Solitary waves}

We start with reproducing the results for solitary waves by using the Euler equations (25)-(26) in the absence of vorticity, which were previously studied in $[10,16,18,47]$. The results are presented in the $c-\zeta(0)$ parametric space as shown in figure 4 . The branch of depression waves is monotonic whereas the one of elevation waves has snake-like behaviour. The bifurcation points are marked as a diamond and a pentagram respectively for the depression and the elevation waves corresponding to the emergence of solitary waves at finite amplitude in figure 3. We extend the elevation branch by further continuing on the curve and explore two-hump solutions which comprises of two depressions (see $(a)$ the lower left graph of figure 4) or two elevations (see (b) the lower right graph of figure 4). The numerical computations terminate at the triangle point which is very close to the pentagram. We plot the associated wave profiles on the bottom right of figure 4. The solution marked with the triangle displays two identical elevation wavepackets glued together in the middle whose the values of $c$ and $\zeta(0)$ are close to those of the pentagram which is a single elevation wavepacket.

We continue by computing solitary waves in the presence of negative vorticity with $\Omega<\Omega^{*}$. The bifurcation diagrams are plotted in figure 5 and 6 for $\Omega=-1$. As expected solitary waves now bifurcate from zero amplitude but otherwise this case displays a qualitatively similar structure as the one presented in figure 4 in the case of no vorticity: the branch of elevation waves still has a snake-like behaviour as shown on the left of figure 5 whereas the depression branch is always monotonic down to $c=0$ in the $c-\zeta(0)$ parametric space. The large depression wave profile of zero velocity is displayed on the right in the same figure. It exhibits no overhanging structure. The $\mathcal{E}-c$ bifurcation diagrams for the elevation and depression waves are sketched in figure 6 . We only present the branches within a range close to $c_{\min }$ to emphasize that the energy of elevation waves in the snaking curve are more simply seen as multiples of that of a depression wave as new packets are "glued" together as in figure 4 (a) and (b).

Next, we work in the case where $\Omega=0.1$, that is, a case where $\Omega>\Omega^{*}$, and follow the solution branches of solitary waves which do not bifurcate from zero. In the $c-\zeta(0)$ parametric space, the branch of depression waves remains monotonic. The branch of elevation waves has zigzag behaviour, but now crosses the minimum phase speed and continuously connects into branches of generalised solitary waves. The numerical computations in figure 7 can become very delicate and were stopped at the point indicated with a triangle. 

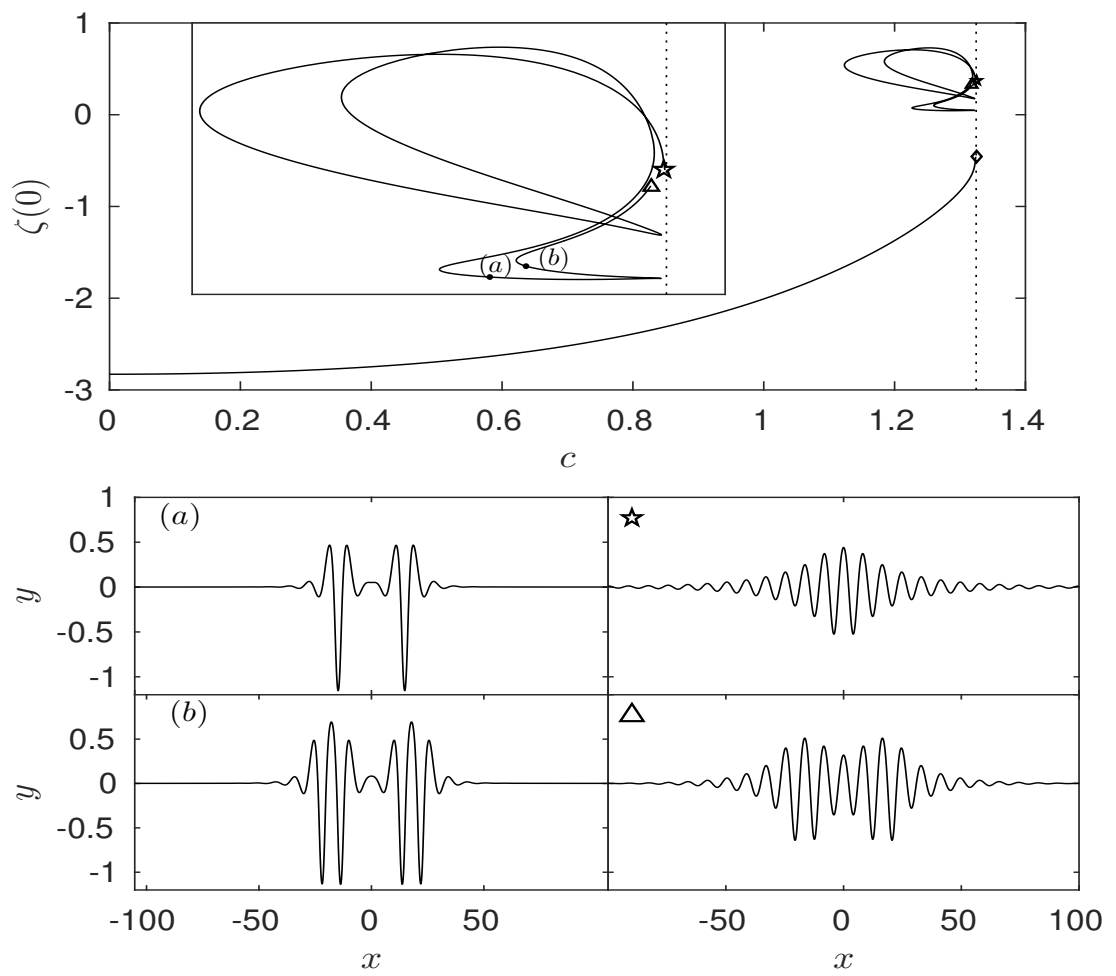

Figure 4: (Top) Global amplitude-speed bifurcation diagrams for $\Omega=0$, i.e. in the absence of vorticity. The upper and lower curves represent elevation and depression waves respectively. The minimum phase speeds are marked as the dotted lines. The diamond is the bifurcation point of the depression wave whereas the pentagram is of the elevation wave. The numerical computation terminates at the triangle point. A blow-up graph near the bifurcation point is presented. Typical wave profiles are sketched.

The curve is expected to continue by snaking across the minimal value of the phase speed. Eight typical wave profiles $(i)-(v i)$, the pentagram and the diamond are displayed in figure 8. Unlike solitary wave whose surface is flat in the far field, $(i)-(v i)$ all have non-decaying oscillating tails in the form of ripples of constant amplitude. These are so-called generalised solitary waves. The branches of Stokes waves bifurcating from the minimum linear speed is also sketched as the dashed-dotted curves in the same picture for comparison. The simplest generalised solitary waves $(i)$, (ii) (with one larger hump in the middle) tend to become periodic waves with all the peaks being of the same amplitude, i.e. approaching the dashed-dotted curves. Figure 9 shows two of the branches in the $\mathcal{E}-c$ plane. (For clarity, only solution branches near the 

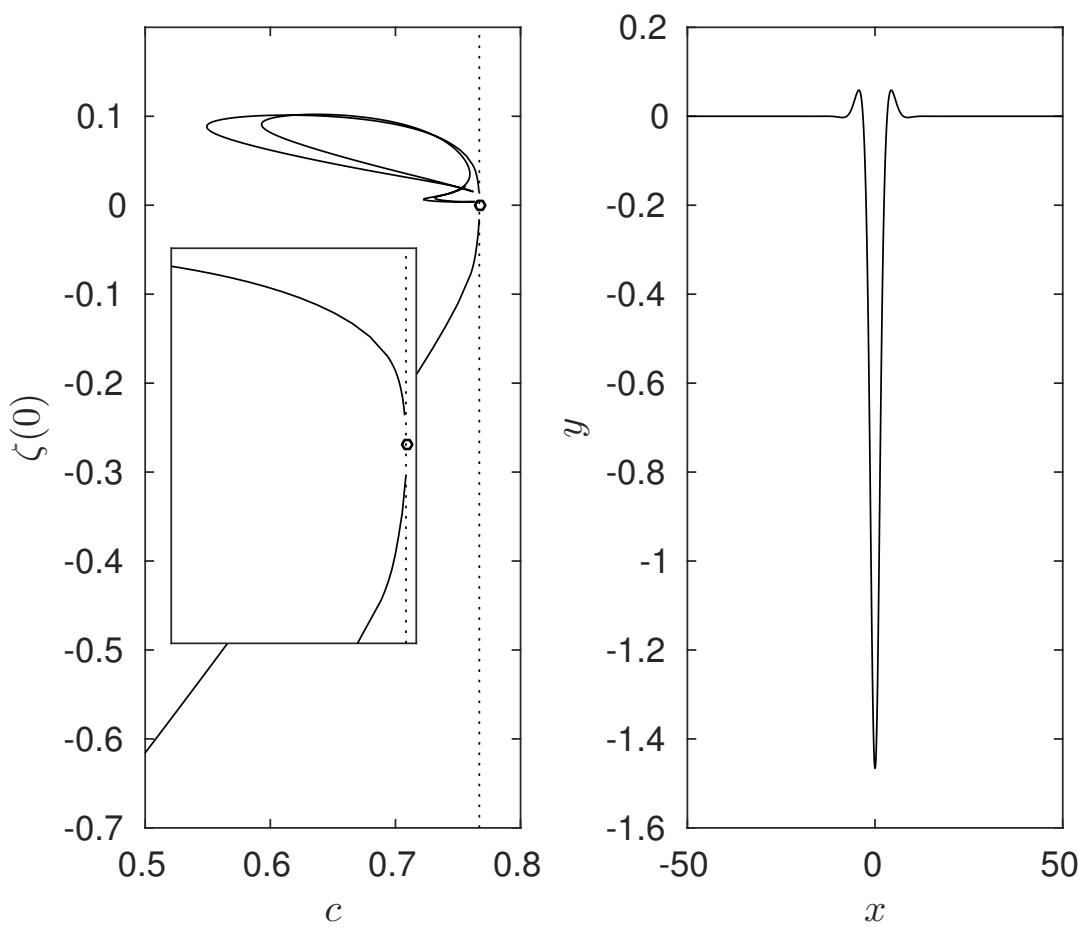

Figure 5: (Left) Global amplitude-speed bifurcation diagrams for $\Omega=-1$. The upper and lower curves represent elevation and depression waves respectively. The numerical computation terminates at the triangle point. (Right) A depression wave profile. The corresponding phase velocity $c$ is zero. The minimum phase speed is marked as the dotted line. Blow-up graphs near the bifurcation point are also drawn.

bifurcation point are presented.) It is important to note that they bifurcate at finite energy and that their energy initially decreases away from the critical speed. There is a minimum of energy at some speed $c<c_{\min }$ and then the energy increases again for even lower $c$. This minimal energy will play a role in the stability of the waves. We note that true generalised solitary waves whose speed $c>c_{\min }$ should have infinite energy due to infinitely many oscillatory ripples in the far field, but our results remain finite as depicted in figure 6 because they are approximated numerically by waves in a finite domain.

Lastly, we investigate asymmetric solitary waves which were considered in [16] and [35] in the absence of vorticity. By using the same numerical procedure, we also find asymmetric solutions. Furthermore, by continuing on 


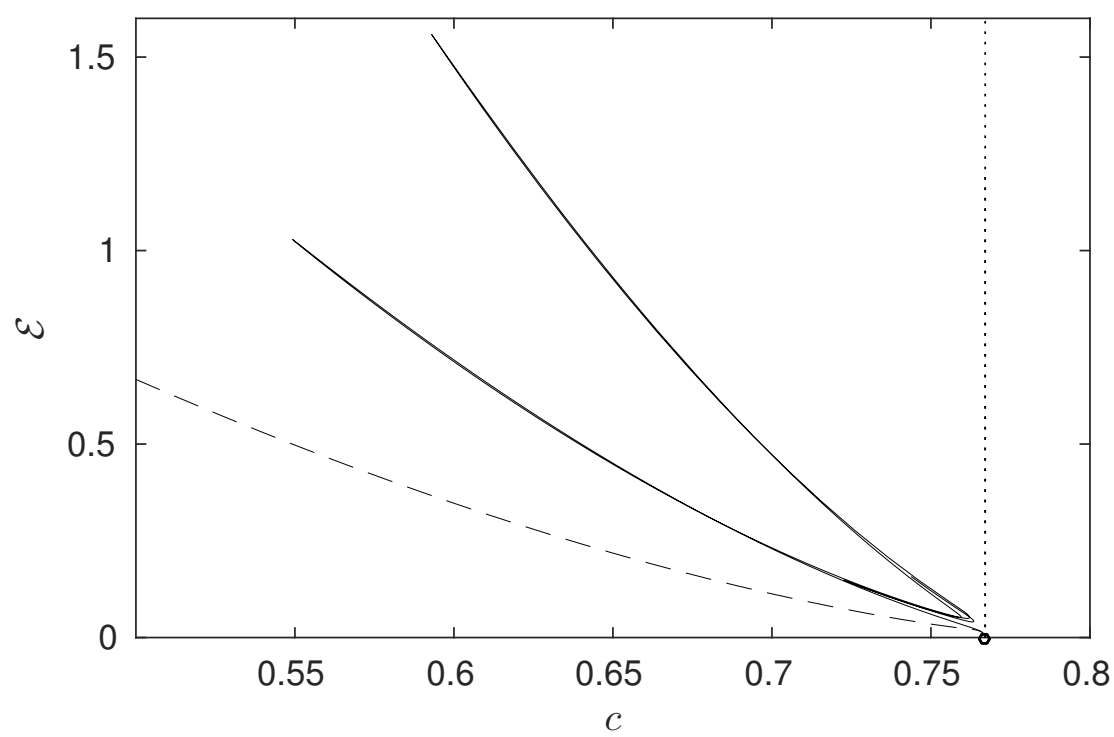

Figure 6: Global energy-speed bifurcation diagrams for the elevation wave (solid curve) and the depression wave (dashed curve) for $\Omega=-1$. The minimum phase speeds is marked as the dotted line.

the solution branch and going over the minimum phase speed, non-decaying ripples in the far field of the wave appear i.e. asymmetric generalised solitary waves. The results are shown in figure 10. Similar solutions were found in the context of electrohydrodynamic free surface waves by [36] by using a quintic model equation. To our knowledge, this is the first time that such waves has been found by using the full Euler equations.

\subsection{Dynamics and Stability of the solitary waves}

In this section, we perform time-dependent simulations on the evolutionary system (22)-(23) for the representative cases chosen in the previous section $\Omega=-1$ and $\Omega=0.1$. We examine numerically the stability and collision of some of the the solitary waves obtained. A frame of reference moving with the speed of one of the waves is usually chosen.

\subsection{1. $\Omega=-1$}

We start with examining the dynamics of a simple elevation wave with a large central hump and decaying tails. We denote this an elevation wave of the first kind for easy reference. This solution is initially perturbed by $1 \%$ 


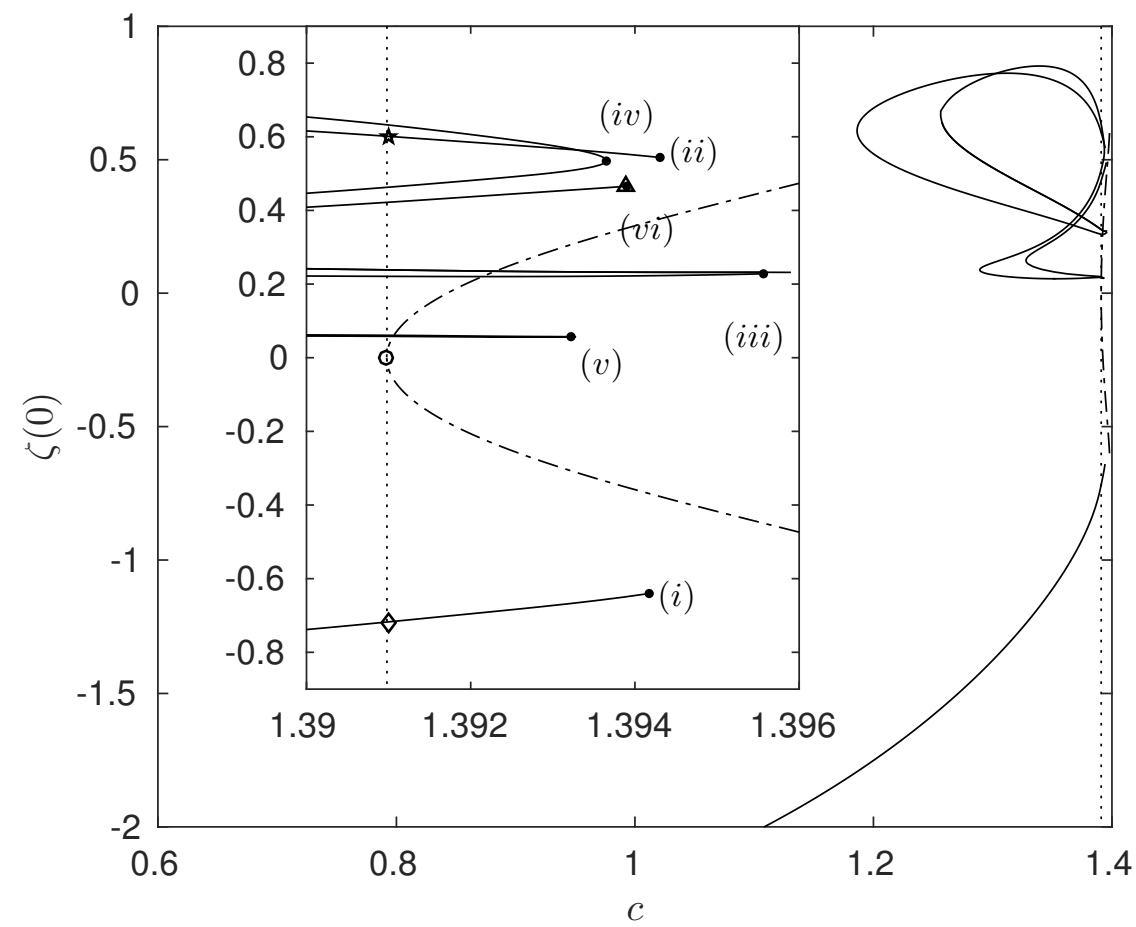

Figure 7: Global amplitude-speed bifurcation diagram of solitary waves for $\Omega=0.1$. The diamond is the bifurcation point of the depression wave and the pentagram is of the elevation wave. The numerical computation terminates at the triangle point which is also marked as point $(v i)$. The dashed-dotted curves represent Stokes waves. They bifurcate at $c_{\text {min }}$ from zero amplitude. A blow-up graph near the bifurcation point is presented.

in amplitude and evolves into a depression wave at later stage. The details are displayed in (1) of figure 11. Similar computations are performed with an elevation wave (2) with two troughs connected by a dimple in the middle (we call it the elevation wave of second kind), and with a depression wave (3). Both are perturbed by $-5 \%$ of the wave amplitude. The waves keep travelling towards the right without losing the shape and no instability has been observed. Other solitary solutions have also been examined but no other stable solutions have been discovered.

Saffman [49] showed that stability exchanges, subject to superharmonic perturbations, of a system with Hamiltonian structure ([50]) can only occur at either stationary or turning points of the energy-speed diagram. Applying the theory to figure 6 , we may conclude that the depression waves are 


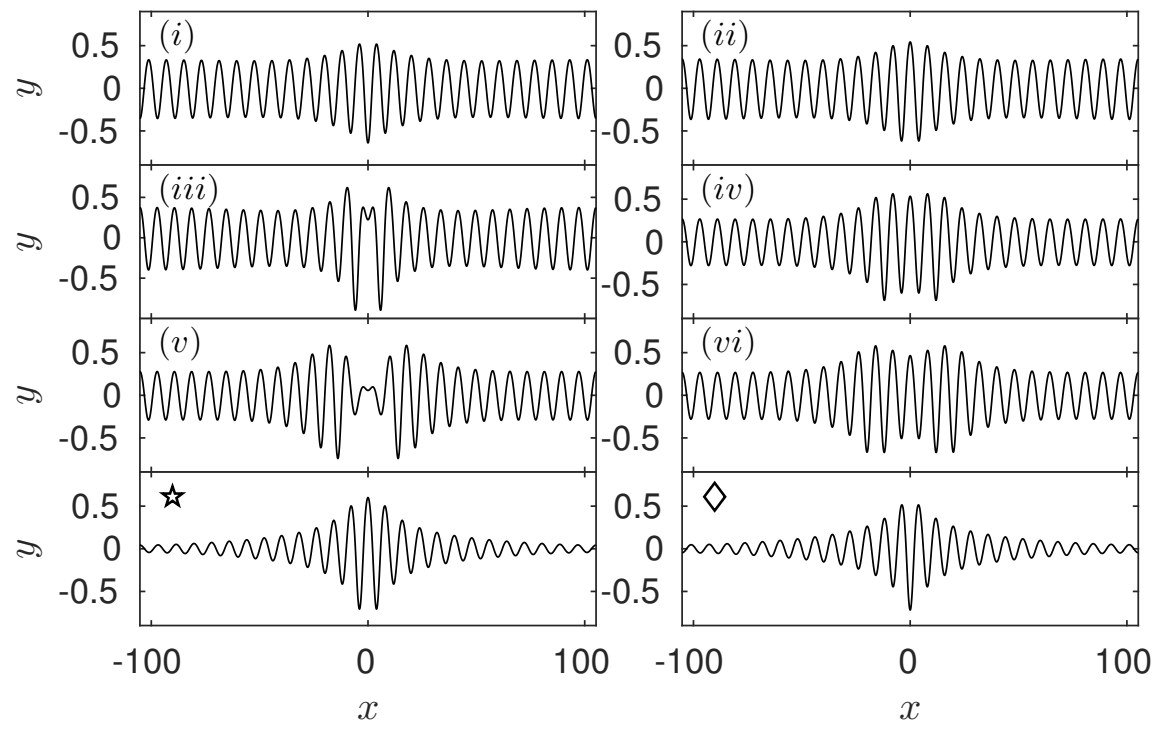

Figure 8: Wave profiles for points $(i)-(v i)$, the pentagram and the diamond marked in figure 7 . Only part of the domain is presented for a better display.

always stable to such perturbations since the branch of the depression waves is monotonic. The stability of the elevation wave has a qualitatively similar scenario to the waves in the irrotational case where stability exchange takes place a few times and only the second kind of elevation wave is stable. The readers are referred to [16] for more details.

\subsection{2. $\Omega=0.1$}

The case where $\Omega=0.1$ is more complex because of the related features of finite amplitude emergence of solitary waves, the presence of generalised solitary waves, and the resultant quintic NLS asymptotic model. We begin with studying the dynamics of solitary waves. The detailed time-dependent computations of five different solitary waves are illustrated in figure 12 and 13 , which are marked as columns (1)- $(4),\left(5^{-}\right)$and $\left(5^{+}\right)$. In column (1) of figure 12 , a depression wave is perturbed by $-5 \%$ of the wave amplitude and is observed to be stable, eventually travelling at a slightly greater speed than originally as it equilibrates to a slightly smaller depression wave (smaller waves travel faster). In column (2)-(4) and $\left(5^{-}\right)$, perturbations of $-1 \%$ of the wave amplitude is applied to solitary waves at $t=0$ whose initial states 

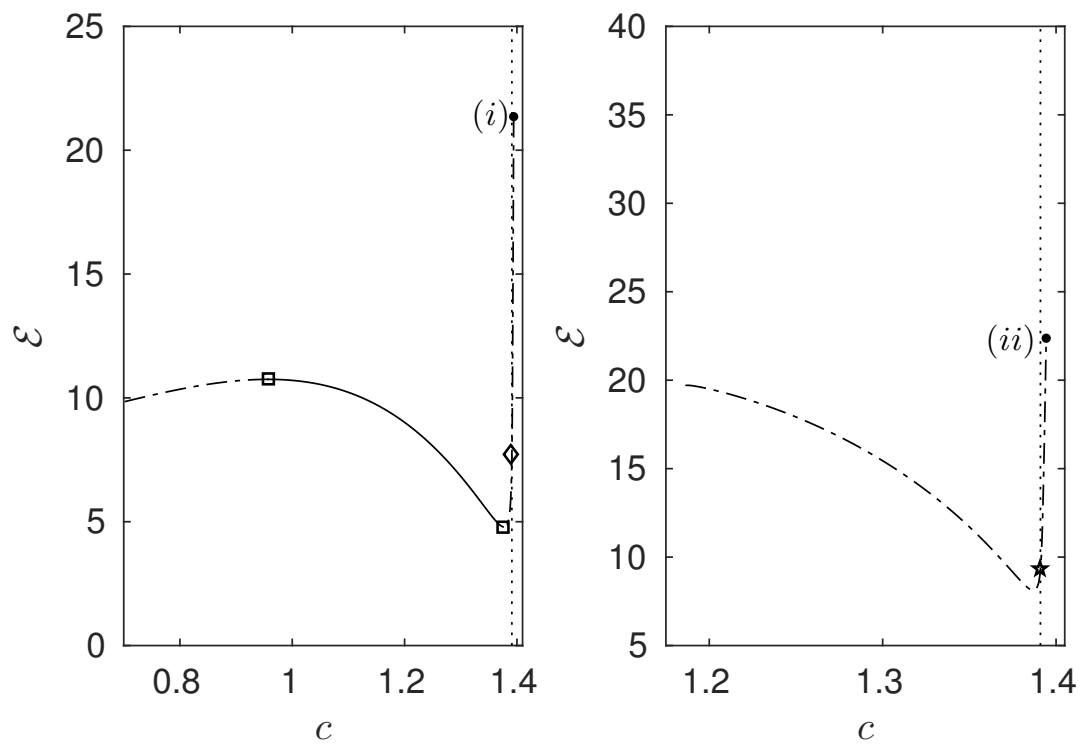

Figure 9: Energy-velocity diagrams for the depression waves (left) and the elevation waves (right) for $\Omega=0.1$. Only part of the two branches are presented for a better display. The dashed-dotted curves are for the unstable solutions. The solid curve is for the stable solutions. The locations of stability exchange are marked as the squares. The diamond, the pentagram, point $(i)$ and $(i i)$ are those presented in figure 7 .

are shown on the top row of the figure. Column (2) displays the dynamics of the first kind of elevation solitary wave. This solution quickly evolves to a stable depression wave along with some radiation of ripples as can be seen on the bottom of column (2). In contrast to the case without constant vorticity [16], the second kind of elevation wave, as shown on the top of (3) in figure 12, is unstable. The asymmetric solitary wave (4) whose left part is an unstable elevation wave unsurprisingly exhibits an instability as the elevation part evolves to a depression wave as time runs on. The dynamics of a depression wave whose propagating speed is close to $c_{\min }$ (and therefore approximated by (31)) is demonstrated in figure 13. The travelling wave solution is initially perturbed by $-1 \%$ in amplitude in column $\left(5^{-}\right)$and $+1 \%$ in $\left(5^{+}\right)$. In the former case, the wave eventually disperses out at $t=$ 500 corresponding to the dispersive spreading phenomenon predicted by the virial theory from section 4.1. Under the effect of an amplitude-increasing perturbation, the wave tends to focus as the mass accumulates near the centre 

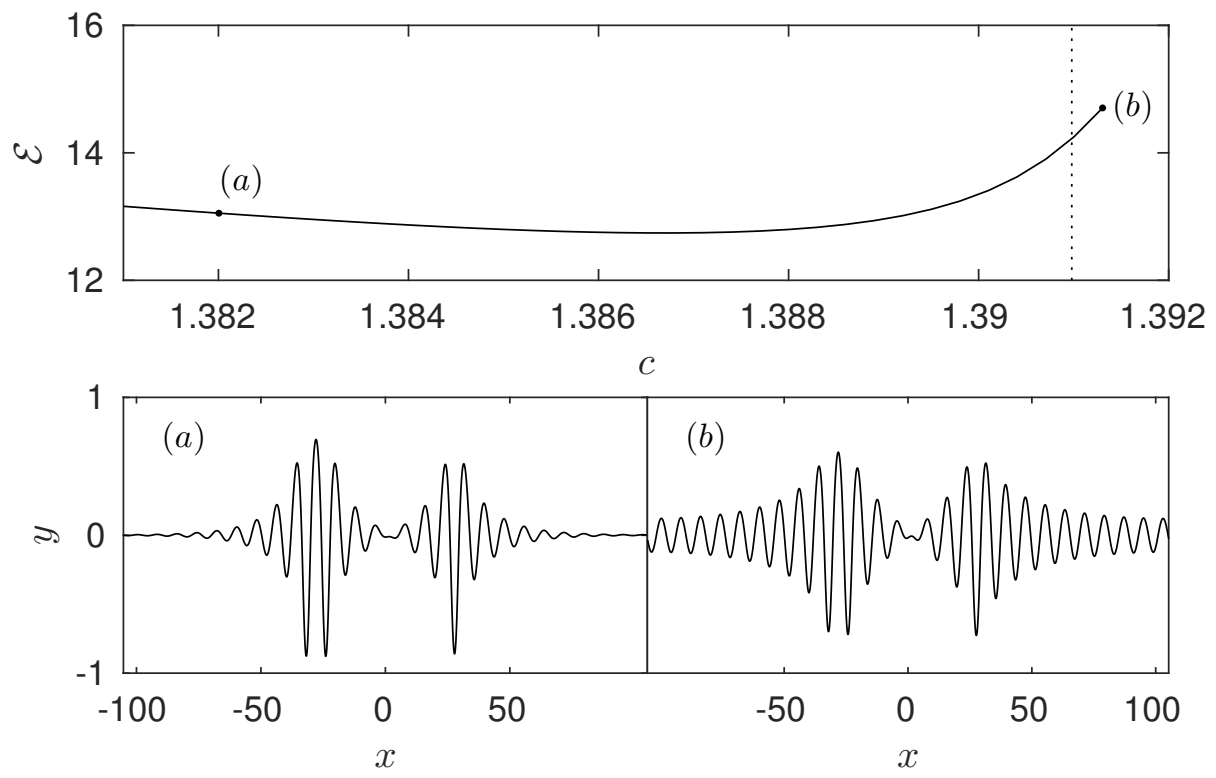

Figure 10: (Top) Local energy-speed bifurcation diagram of the asymmetric solitary waves. $c_{\text {min }}$ is sketched as the dotted line. (Bottom) Wave profiles which correspond to $(a)$ and (b) from the upper graph. All the wave profiles are sketched in the physical plane. Only part of the domain is plotted for a better display.

of the wavepacket as can be seen from the snapshots at $t=125$ and $t=250$. However the full collapsing phenomenon predicted by the virial theory does not take place since the solution converges to a stable depression wave from the stable branch as shown in figure 9 with a larger amplitude and a slower propagating speed in comparison to the initial state. virial theory ceases to predict the evolution correctly after some time, as that is a result for the quintic NLS equation and therefore only applies to the Euler equations approximately. The stabilities of the depression waves are summarised in the left graph of figure 9 where stability exchange occurs twice. The dotteddashed curves are for the unstable solutions and the solid one is for the stable.

We now turn to generalised solitary waves. From virial theory it is expected that generalised solitary waves are also unstable, as the branches are a continuation from unstable solitary waves. We verify this claim by performing numerical examinations where $-1 \%$ perturbations in amplitude are initially applied to the generalised depression and elevation waves, which can 

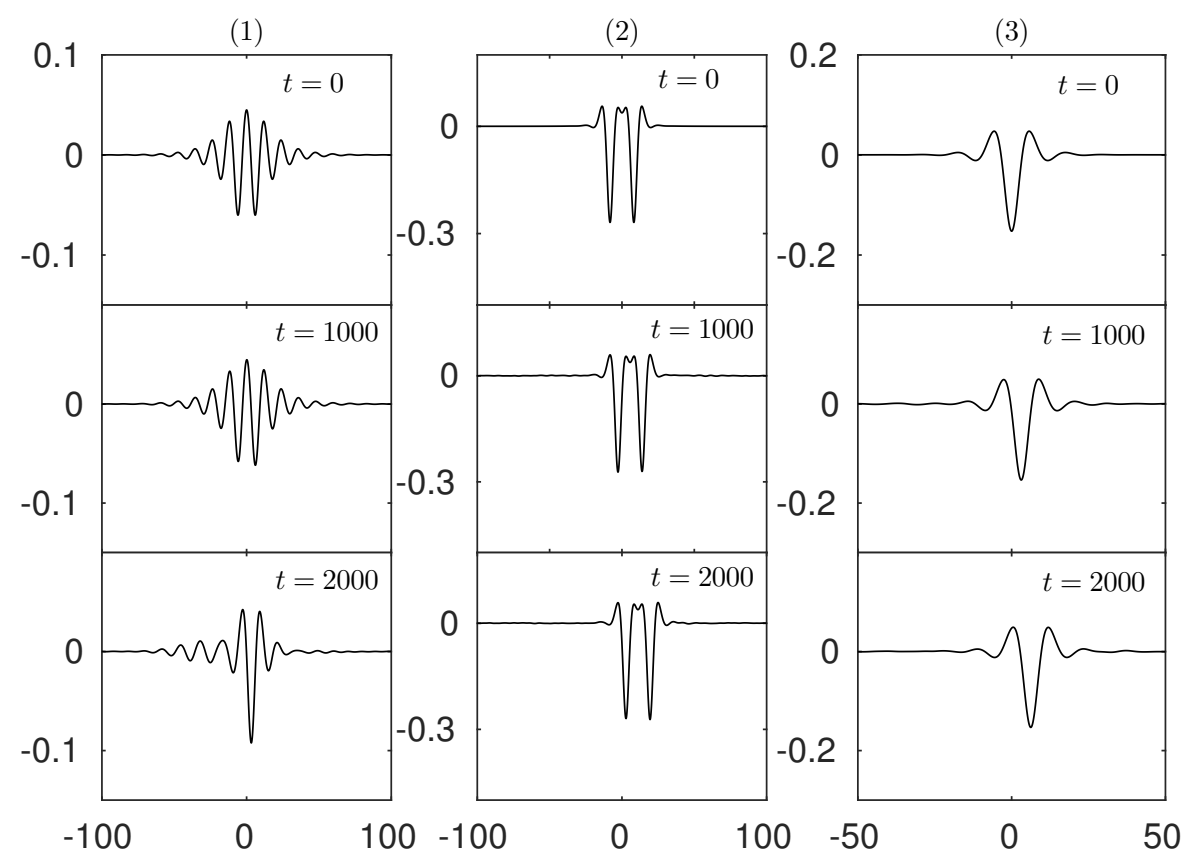

Figure 11: Dynamics of solitary waves for $\Omega=-1$. The corresponding values of $c$ are (1) 0.7617, (2) 0.6751 and (3) 0.7300. All the snapshots are plotted in the physical plane.

be achieved by choosing a large value of domain size $L$ such that the periodic boundary condition does not have much effect to the central part of the wave. The dynamics of the generalised depression wave is quite simple as can be seen in (7) (the middle column) of figure 14 where it eventually disperses out at $t=500$. The evolution of the generalised elevation wave is more similar to the collapse dynamics (see (6) of figure 14) as mass focusses into generalised depression wave together with a modulation of the finite amplitude tails of the generalised wave. The asymmetric generalised solitary wave has the same nature of instability, as seen in our time-dependent computation (see (8) in figure 14). The dynamics of this solution is similar to (4) from figure 12 where the elevation part becomes depression some time after the initial perturbation. Solutions $(a)$ and $(b)$ in figure 10 are both unstable and therefore the asymmetric branch presented there expected to be unstable everywhere. We come to a conclusion that only the depression waves from the solid curve of figure 9 are stable and all the other solutions are unstable. 


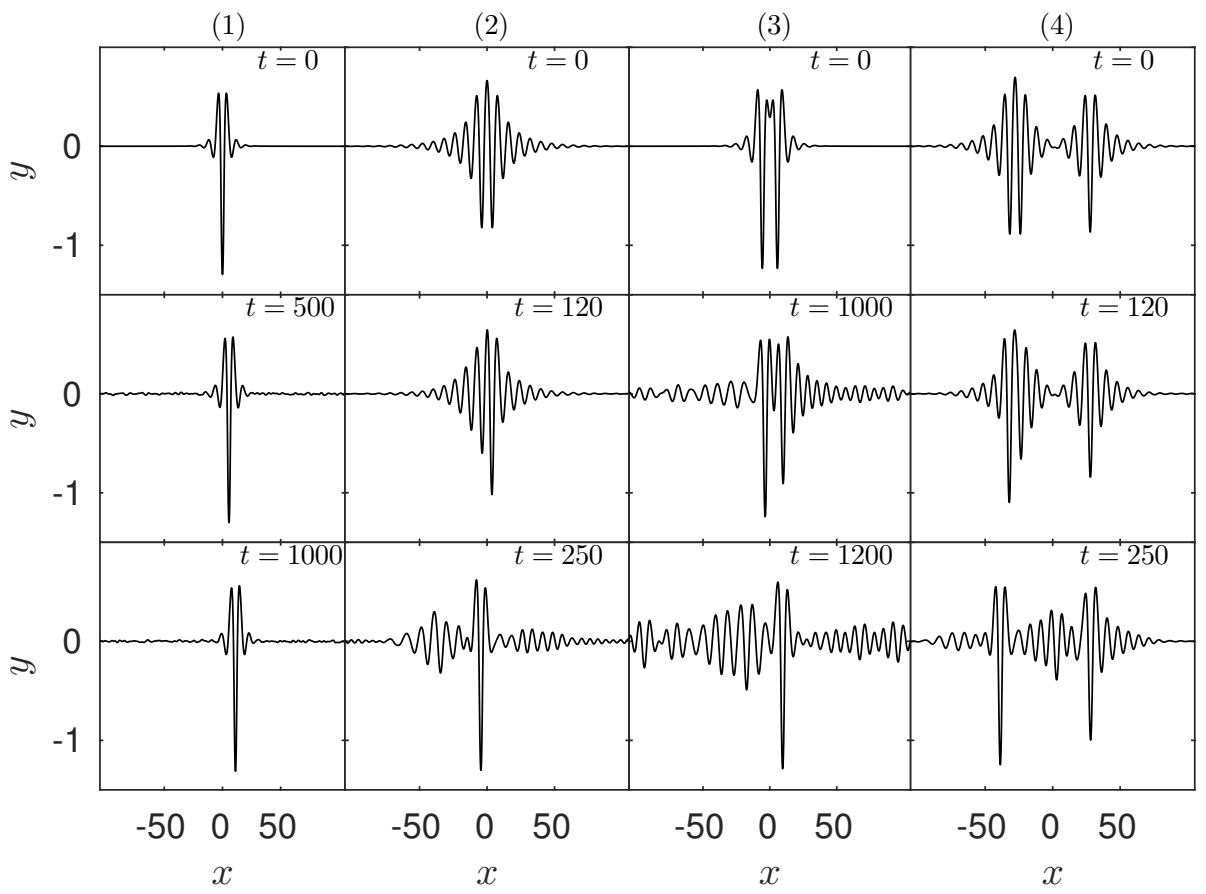

Figure 12: Dynamics of the solitary waves for $\Omega=0.1$ where an amplitude-decreasing perturbation is applied at $t=0$. The corresponding initial propagating speeds are $(1)$ 1.3028, (2) 1.3828, (3) 1.3381 and (4) 1.3809.

\subsection{Head-on collision}

In this section we perform a numerical experiment of head-on collision between two stable depression waves from two different families. We choose $\Omega=0.1$ and $c=1.23$ for the wave travelling rightwards. For the left-moving wave, we need to compute the solution for $\Omega=-0.1$ which is therefore from a different regime (ie - focussing or defocussing at the cubic level) as can bee seen from figure 2. We choose $c=-1.19$ for the left-going wave and the following numerical parameters: $L=400, N=4096, d t=0.00025$. The initial state is set by superposing two shifted depression waves as presented on top of figure 15. We present the head-on collision by sketching the snapshots of the dynamics before the interaction at $t=0,70$ and after the interaction at $t=100,125$. One can observe an inelastic collision with substantial energy radiation in the form of small ripples after the collision. 


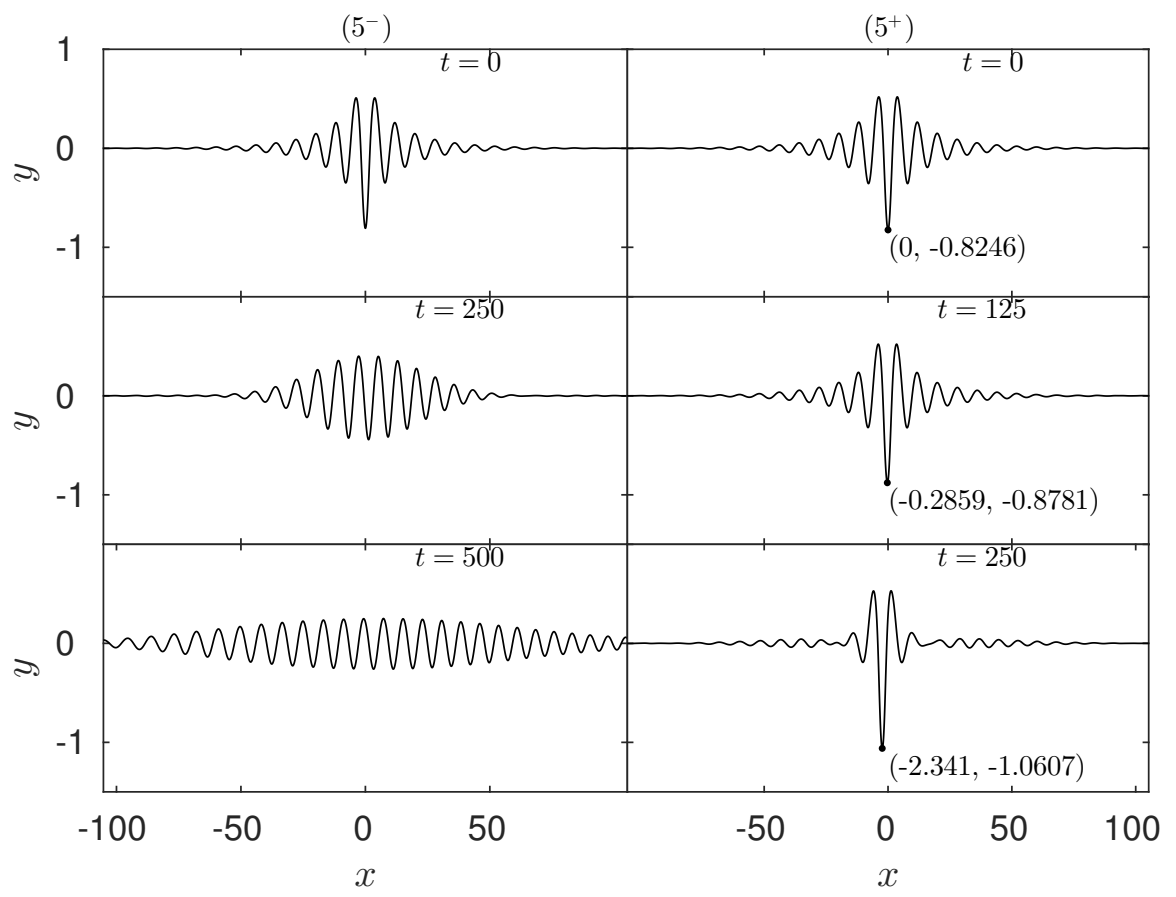

Figure 13: Dynamics of a solitary wave with initial propagating speed 1.3850 for $\Omega=0.1$. At $t=0$ the wave amplitude is perturbed by $-1 \%$ in $\left(5^{-}\right)$and $+1 \%$ in $\left(5^{+}\right)$.

\section{Conclusion}

In this work, the problem of hydroelastic waves in the presence of constant vorticity was studied. A high-order Nonlinear Schrödinger Equation was presented to investigate whether a given envelope collapses into a singular point in finite time by using virial theory. A time-dependent conformal map technique was used to solve the fully nonlinear problem in both the steady and unsteady cases. Two branches of steady solitary waves were computed for $\Omega=-1,0,0.1$. The complete bifurcation diagrams for different values of $\Omega$ were presented. The numerical results showed that the solitary waves bifurcate from zero amplitude for $\Omega=-1$ while from finite amplitude for $\Omega=0$ and $\Omega=0.1$. Asymmetric solitary wave was found by following the mechanism introduced in [35]. New generalised solitary waves were discovered for $\Omega=0.1$. The stabilities of these solutions, subject to amplitude perturbations, were examined numerically by using the scheme introduced in section 3. A head-on collision between two stable depression solitary waves belonging to different branches was performed. 


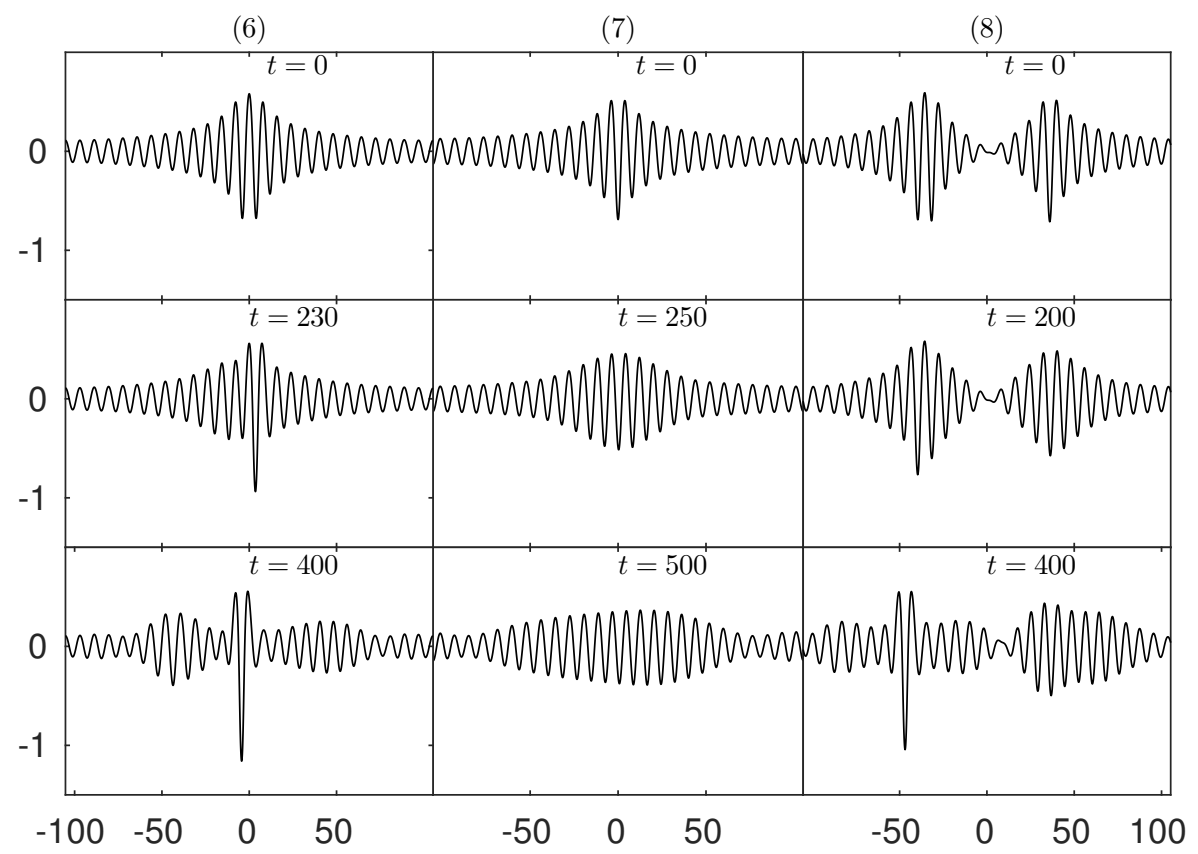

Figure 14: Dynamics of the generalised solitary waves for $\Omega=0.1$. The corresponding initial propagating speeds are (6) 1.3919 , (7) 1.3920 and (8) 1.3913. All the snapshots are plotted in the physical plane. Only part of the domain is shown.

\section{Acknowledgement}

J.-M.V.-B. acknowledges the support from EPSRC Grant No. EP/N018559/1. P.M. was supported by EPSRC under Grant No. EP/N018176/1.

[1] V. A. Squire, J. P. Dugan, P. Wadhams, P. J. Rottier, \& A. K. Liu, Of ocean waves and sea ice. Ann. Rev. Fluid Mech. 27(1) (1995) 115-168.

[2] V. A. Squire, W. Robinson, P. Langhorne, T. Haskell, Vehicles and aircraft on floating ice, Nature 333 (6169) (1988) 159-161.

[3] G. D. Ashton, River and lake ice engineering, Water Resources Publication, 1986.

[4] V. A. Squire, R. J. Hosking, A. D. Kerr, P. J. Langhorne, Moving loads on ice plates (solid mechanics and its applications) (1996). 


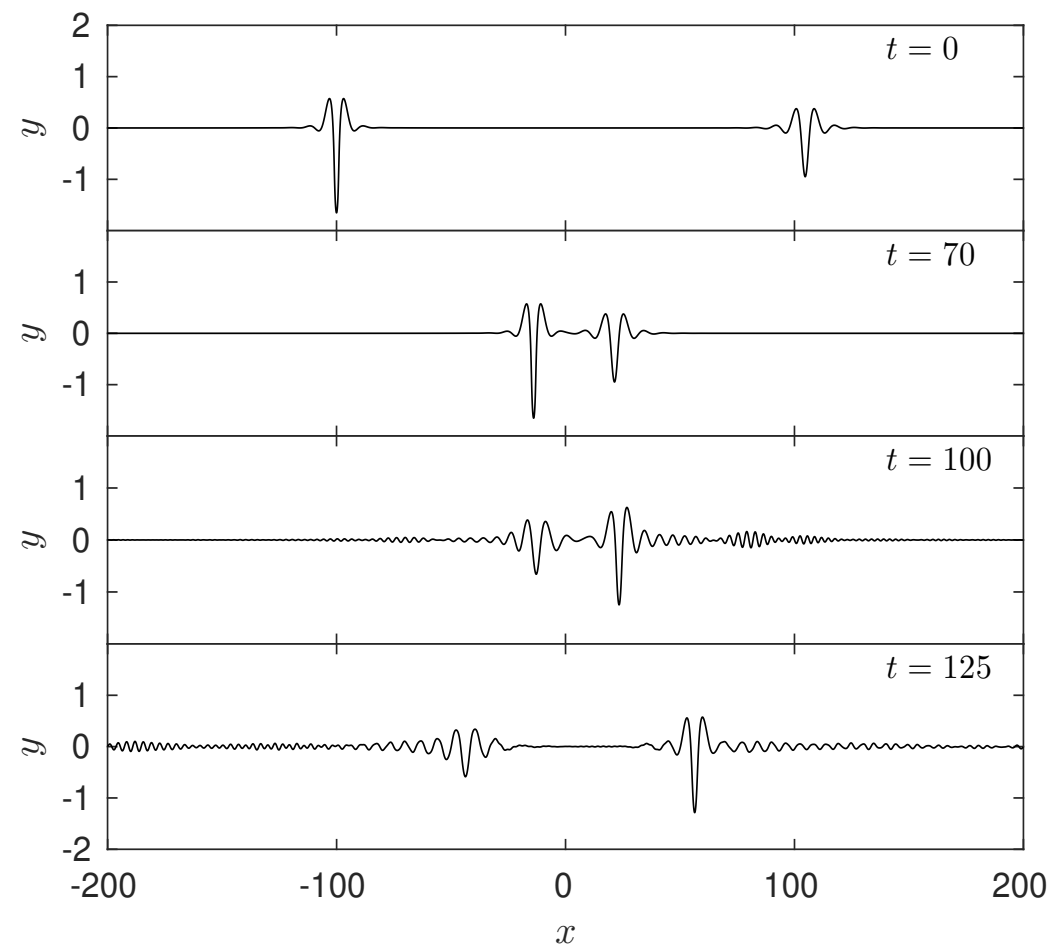

Figure 15: Head-on collision of two depression waves when $\Omega=0.1$. The one with larger amplitude is travelling rightwards with $c=1.23$ while the smaller one is moving leftwards with speed $c=1.19$.

[5] T. Takizawa, Deflection of a floating sea ice sheet induced by a moving load, Cold Reg. Sci. Technol. 11 (2) (1985) $171-180$.

[6] L. K. Forbes, Surface waves of large amplitude beneath an elastic sheet. part 1. high-order series solution, J. Fluid Mech. 169 (1986) 409-428.

[7] L. K. Forbes, Surface waves of large amplitude beneath an elastic sheet. part 2. galerkin solution, J. Fluid Mech. 188 (1) (1988) 491-508.

[8] J.-M. Vanden-Broeck, E. I. Părău, Two-dimensional generalized solitary waves and periodic waves under an ice sheet, Philos. Trans. Royal Soc. A 369 (1947) (2011) 2957-2972.

[9] P. A. Milewski, J.-M. Vanden-Broeck, Z. Wang, Steady dark solitary flexural gravity waves, Proc. R. Soc. A 469 (2150) (2013) 20120485. 
[10] P. A. Milewski, J.-M. Vanden-Broeck, Z. Wang, Hydroelastic solitary waves in deep water, J. Fluid Mech. 679 (2011) 628-640.

[11] F. Bonnefoy, M. H. Meylan, P. Ferrant, Nonlinear higher-order spectral solution for a two-dimensional moving load on ice, J. Fluid Mech. 621 (2009) 215-242.

[12] E. I. Părău, F. Dias, Nonlinear effects in the response of a floating ice plate to a moving load, J. Fluid Mech. 460 (2002) 281-305.

[13] J. Toland, Heavy hydroelastic travelling waves, Proc. R. Soc. A 463 (2085) (2007) 2371-2397.

[14] J. Toland, Steady periodic hydroelastic waves, Arch. Ration. Mech. Anal. 189 (2) (2008) 325-362.

[15] T. Gao, J.-M. Vanden-Broeck, Numerical studies of two-dimensional hydroelastic periodic and generalised solitary waves, Phys. Fluids 26 (8) (2014) 087101.

[16] T. Gao, Z. Wang, J.-M. Vanden-Broeck, New hydroelastic solitary waves in deep water and their dynamics, J. Fluid Mech. 788 (2016) 469-491.

[17] P. Guyenne, E. I. Părău, Finite-depth effects on solitary waves in a floating ice sheet, J. Fluids Struct. 49 (2014) 242-262.

[18] P. Guyenne, E. I. Părău, Computations of fully nonlinear hydroelastic solitary waves on deep water, J. Fluid Mech. 713 (2012) 307-329.

[19] Constantin, A., Strauss, W. A. Stability properties of steady water waves with vorticity. Comm. Pure Appl. Math., 60(6), (2007) 911-950.

[20] J. A. Simmen, P. G. Saffman, Steady Deep-Water Waves on a Linear Shear Current, Stud. Appl. Math. 73 (1) (1985) 35-57.

[21] A. F. Da Silva Teles, D. H. Peregrine, Steep, steady surface waves on water of finite depth with constant vorticity, J. Fluid Mech. 195 (1988) 281-302.

[22] J.-M. Vanden-Broeck, Steep solitary waves in water of finite depth with constant vorticity, J. Fluid Mech. 274 (1994) 339-348. 
[23] J.-M. Vanden-Broeck, Periodic waves with constant vorticity in water of infinite depth, IMA J. Appl. Math. 56 (3) (1996) 207-217.

[24] J.-M. Vanden-Broeck, New families of steep solitary waves in water of finite depth with constant vorticity, Eur. J. Mech. B Fluids, 14 (6) (1995) 761-774.

[25] Y. Kang, J.-M. Vanden-Broeck, Gravity-capillary waves in the presence of constant vorticity, Eur. J. Mech. B Fluids, 19 (2) (2000) 253-268.

[26] W. Choi, Nonlinear surface waves interacting with a linear shear current, Math. Comput. Simulat. 80 (1) (2009) 29-36.

[27] O. G. Nwogu, Interaction of finite-amplitude waves with vertically sheared current fields, J. Fluid Mech. 627 (2009) 179-213.

[28] M. Francius, C. Kharif, Two-dimensional stability of finite-amplitude gravity waves on water of finite depth with constant vorticity, J. Fluid Mech. 830 (2017) 631-659.

[29] D. Guo, B. Tao, X. Zeng, On the dynamics of two-dimensional capillarygravity solitary waves with a linear shear current, Adv. Math. Phys. (2014).

[30] P. R. Garabedian, Surface waves of finite depth. J. d'Anal. Math. 14, (1965) 161-169.

[31] J. F. Toland, On the symmetry theory for Stokes waves of finite and infinite depth. Trends in applications of mathematics to mechanics 106, (2000) 207-217.

[32] A. Constantin, , J. Escher, Symmetry of steady periodic surface water waves with vorticity. J. Fluid Mech. 498, (2004) 171-181.

[33] A. Constantin, , J. Escher, Symmetry of steady deep-water waves with vorticity. Eur. J. Appl. Math. 15(6), (2004) 755-768.

[34] T.-S. Yang, T. R. Akylas, On asymmetric gravity-capillary solitary waves, J. Fluid Mech. 330 (1997) 215-232.

[35] Z. Wang, J.-M. Vanden-Broeck, P. Milewski, Asymmetric gravitycapillary solitary waves on deep water, J. Fluid Mech. 759 (2014). 
[36] Z. Lin, Y. Zhu, Z. Wang, Local bifurcation of electrohydrodynamic waves on a conducting fluid, Phys. Fluids 29 (3) (2017) 032107.

[37] Z. Wang, P. A. Milewski, Dynamics of gravity-capillary solitary waves in deep water. J. Fluid Mech., 708 (2012) 480-501.

[38] T. Gao, Z. Wang, J.-M. Vanden-Broeck, On asymmetric generalized solitary gravity-capillary waves in finite depth, Proc. R. Soc. A 472 (2194) (2016) 20160454.

[39] A. I. Dyachenko, V. E. Zakharov, E. A. Kuznetsov, Nonlinear dynamics of the free surface of an ideal fluid, Plasma Phys. Rep. 22 (10) (1996) 829-840.

[40] W. Choi, R. Camassa, Exact evolution equations for surface waves, J. Eng. Mech. 125 (7) (1999) 756-760.

[41] Y. A. Li, J. M. Hyman, W. Choi, A numerical study of the exact evolution equations for surface waves in water of finite depth, Stud. Appl. Math. 113 (3) (2004) 303-324.

[42] R. Ribeiro, P. A. Milewski, A. Nachbin, Flow structure beneath rotational water waves with stagnation points. J. Fluid Mech., 812, (2017) 792-814.

[43] Shabat, A., Zakharov, V. Exact theory of two-dimensional self-focusing and one-dimensional self-modulation of waves in nonlinear media. Soviet physics JETP, 34(1), (1972) 62.

[44] Milewski, P. A., \& Wang, Z. Self-focusing dynamics of patches of ripples. Physica D, 333, (2016) 235-242.

[45] Rasmussen, J. J., \& Rypdal, K. Blow-up in Nonlinear Schröedinger Equations - I a general review. Phys. Scr., 33(6), (1986) 481.

[46] Sulem, C., \& Sulem, P. L. The Nonlinear Schrödinger equation: selffocusing and wave collapse. Springer Science \& Business Media, 2007.

[47] Z. Wang, J.-M. Vanden-Broeck, P. Milewski, Two-dimensional flexuralgravity waves of finite amplitude in deep water, IMA J. Appl. Math. 78(4), (2013) 750-761. 
[48] T. Gao, Z. Wang, P. A. Milewski, Nonlinear hydroelastic waves on a linear shear current at finite depth. (submitted)

[49] P. Saffman, The superharmonic instability of finite-amplitude water waves, J. Fluid Mech. 159 (1985) 169-174.

[50] V. E. Zakharov, Stability of periodic waves of finite amplitude on the surface of a deep fluid, J. Appl. Mech. Tech. Phys. 9 (2) (1968) 190-194. 